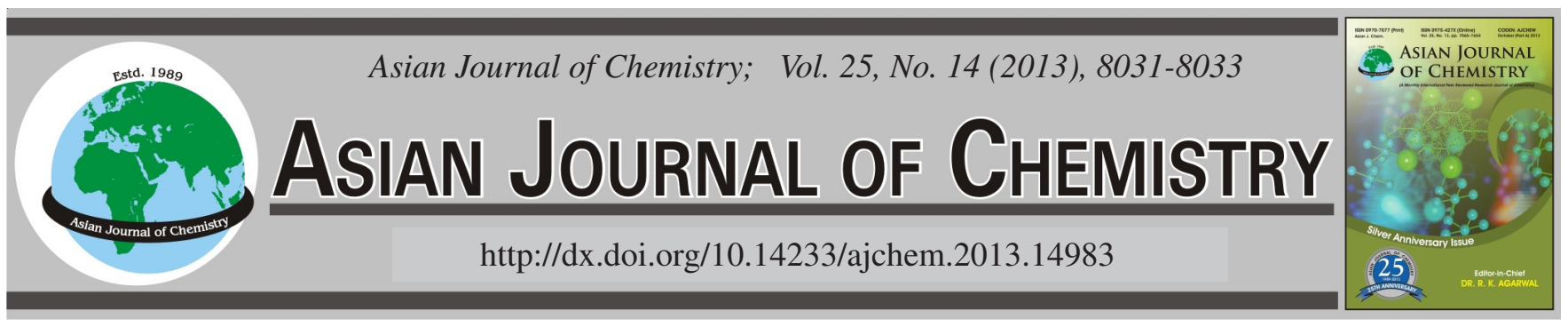

\title{
Poly(sulfobetaine methacrylate)-Modified \\ Dimethylpolysiloxane with Improved Antibiofouling Property
}

\author{
Fengling Xu*, Cunguo Lin, Jinwei Zhang, Jiyong Zheng, Li Wang and Zhiyong Sun
}

State Key Laboratory for Marine Corrosion and Protection; Luoyang Ship Material Research Institute, 149 Zhuzhou Road, Qingdao 266101, P.R. China

*Corresponding author: Fax: +86 532 68725001; Tel: +86 532 68725028; E-mail: xufl@ sunrui.net; xufengling1980@yahoo.com.cn

\begin{abstract}
This paper describes a facile and novel approach for the surface modification of dimethylpolysiloxane membranes by introducing poly(sulfobetaine methacrylate) groups grafted via atom transfer radical polymerization (ATRP) for their resistance to protein-adsorption and diatom-adsorption. Chemical changes of the membrane surface were characterized by attenuated total reflectance Fourier transform infrared spectroscopy (ATR/FT-IR). Results revealed that poly(sulfobetaine methacrylate) groups have been successfully introduced to the dimethylpolysiloxane membrane surface. The surface properties of membranes were characterized by water-contact angle and protein adsorption measurements. It was found that the hydrophilicity was effectively improved and the bovine serum albumin adsorption was significantly suppressed by introducing poly(sulfobetaine methacrylate) groups. Finally, the diatom bioassays were measured further to evaluate the antifouling property for the studied membranes. Results showed that poly(sulfobetaine methacrylate)-modified dimethylpolysiloxane membranes had higher diatom-adsoption-resistance property. So the poly(sulfobetaine methacrylate)-modified dimethylpolysiloxane membranes have improved protein-adsorption-resistance and diatom-adsorption-resistance properties and should be potential marine antibiofouling materials.
\end{abstract}

Key Words: Dimethylpolysiloxane, Poly(sulfobetaine methacrylate), Antibiofouling.

\section{INTRODUCTION}

Fouling of natural and artificial structures is a serious problem in the marine environment resulting in vast economic losses. While fouling organisms are considered a nuisance to ships, buoys and other floating structures, as well as to pilings in harbours, they may cause serious inefficiencies to sound devices, electronic units, cables and other instruments intended for underwater research. It is found that the initial step in biofouling formation is the adsorption of protein that the marine biology secreted to substratum surfaces. It is found that the mussel adhesion is mediated by the byssus and much research has focussed on characterizing those byssal proteins which containing Mytilus edulis food protein 1 (Mefp-1), Mefp-2, Mefp-3 and so on ${ }^{1}$. Because of its highly adsorptive and surface-active behaviour in vitro ${ }^{2-4}$, Mefp-1 has long been regarded as a key ingredient of mussel adhesion. Lind et al. ${ }^{5}$ found that substratum adhesion and gliding in a diatom were mediated by extracellular proteoglycans. Many other researches found that the adsorption of barnacle based on a kind of colloid secreted by barnacle secreting which mainly composed by protein. So it is important and significant for antifouling to inhibit the adsorption of protein.
It is found that surfaces that resist the adsorption of proteins incorporate groups that exhibit four molecular-level characteristics: (i) They are hydrophilic. (ii) They include hydrogen-bond acceptors. (iii) They do not include hydrogenbond donors. (iv) Their overall electrical charge is neutral ${ }^{6}$. It was reported in the literature that poly(sulfobetaine methacrylate) $[\mathrm{p}(\mathrm{SBMA})]$ is highly resistant to protein and bacterial adsorption and can dramatically reduced biofilm formation ${ }^{7}$. Whereas here we describes a facile and novel approach for the surface modification of dimethylpolysiloxane (PDMS) membranes by introducing $\mathrm{p}$ (SBMA) groups grafted via atom transfer radical polymerization (ATRP) for their resistance to protein-adsorption and diatom-adsorption, as schematically described in Scheme-I.

\section{EXPERIMENTAL}

Dimethylpolysiloxane (PDMS), bromoisobutyryl bromide (BIBB $98 \%$ ), N-(3-sulfopropyl)-N-(methacryloxyethyl)-N,Ndimethylammonium betaine (SBMA $97 \%$ ), 2,2-bipyridine (BPY $99 \%$ ), $\mathrm{CuBr}$ and $0.15 \mathrm{M}$ phosphate-buffered saline (PBS) were purchased from Sigma-Aldrich (Milwaukee, USA). Bovine serum albumin (BSA, Mw $=68,000$ ) was purchased from Amresco (American). 


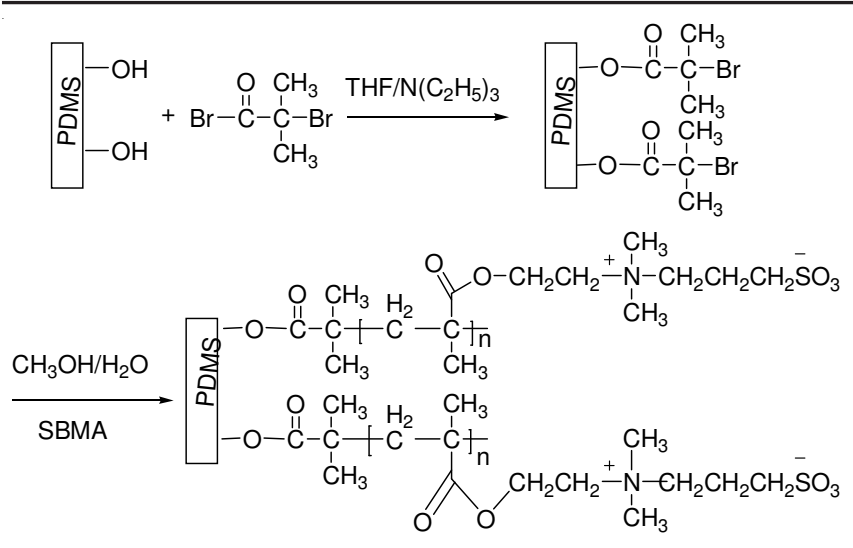

Scheme-I: Schematic representative for the preparation of poly(SBMA)modified PDMS membranes

Preparation of PDMS-Br initiator monolayers: In this study initiator bromoisobutyrate monolayers was formed on the substrates. For the preparation of initiator monolayers on PDMS surface, hydroxyl was first introduced to the original PDMS surface by dielectric barrier discharge. And then the PDMS-OH chip was soaked in a stirred mixture solution of triethylamine and tetrahydrofuran in $250 \mathrm{~mL}$ rockered flask with ice bath. $1 \mathrm{mmol}$ bromoisobutyrate was added. After 5 min, withdraw the ice bath. The reaction was continually carried for $16 \mathrm{~h}$ to form initiator monolayers on the PDMS surface and the chip was rinsed with pure ethanol and then dried in a stream of $\mathrm{N}_{2}$.

Preparation of $\mathrm{p}(\mathrm{SBMA})$ modified PDMS surface: $\mathrm{p}$ (SBMA) modified PDMS surface were achieved via the surface-initiated ATRP method and were prepared following the method reported previously ${ }^{8}$. $\mathrm{p}(\mathrm{SBMA})$ brushes were polymerized on the PDMS-Br substrates with immobilized initiators of Br-SAMs. The reaction solution of $\mathrm{CuBr}$ and 2,2'bipyridine were first placed in a sealed glass reactor in a dry box under a nitrogen atmosphere. A $10 \mathrm{mM}$ degassed solution (pure water and methanol in a 1:3 volume ratio) of SBMA monomers was transferred to the reactor and the PDMS surface with immobilized initiators was then placed in the reactor under nitrogen. After polymerization, the p(SBMA)-modified PDMS chip was removed and rinsed with ethanol and water and the samples were kept in water overnight. The prepared substrates were usually rinsed with PBS buffer to remove unbound polymers before any experiments.

\section{RESULTS AND DISCUSSION}

Fabrication of pSBMA modified PDMS membranes: In this work, $\mathrm{p}$ (SBMA) were anchored on the PDMS membrane surface by chemical covalent bonds. THF was chosen as the reaction medium because in the polar solvent the hydroxyl groups of PDMS surface can relatively easily migrate to the top surface of the membrane and in turn benefits the reaction of the hydroxyl group with bromoisobutyrate. To ensure high conversion of hydroxyl groups on the surface into $\mathrm{p}$ (SBMA) moieties, membrane must be completely dried before the reaction. And the amount of SBMA were excessive than that of the bromoisobutyrate groups on the membrane surface, which could ensure most of the bromoisobutyrate groups on the membrane surface be converted into $\mathrm{p}$ (SBMA) groups.
The original and modified PDMS membranes were characterized by ATR/FT-IR spectroscopy. Compared with the FTIR spectra of original PDMS, the FT-IR spectra of $\mathrm{p}$ (SBMA)PDMS have two new absorbance peaks at 1716 and $835 \mathrm{~cm}^{-1}$, which are respectively attributed to the stretching vibration of $\mathrm{C}=\mathrm{O}$ and $\mathrm{S}-\mathrm{O}$. However, the absorbance peaks of $-\mathrm{N}^{+}\left(\mathrm{CH}_{3}\right)_{2}-$ group and $\mathrm{S}=\mathrm{O}$, which is at $c a .967 \mathrm{~cm}^{-1}$, is covered up by the absorbance peak of C-O $\left(1300-900 \mathrm{~cm}^{-1}\right)$ in the vicinity and cannot be discerned.

Hydrophilicity properties: The hydrophilicity of studied membranes was characterized on the basis of water-contact angle measurements. The static water-contact angles of original PDMS and p(SBMA) modified PDMS surfaces were measured by the sessile drop method using a contact angle goniometer (Dataphysics OCA15, Germany). Droplets of distilled water $(2 \mu \mathrm{L})$ were dropped at different places of the membranes and at least 10 readings were taken to determine average values. As presented in Fig. 1, the contact angles on the p(SBMA)PDMS membranes were obviously lower than those on the PDMS membranes, which proved that the hydrophilicity was effectively improved by introducing $\mathrm{p}$ (SBMA) onto the membrane surface.

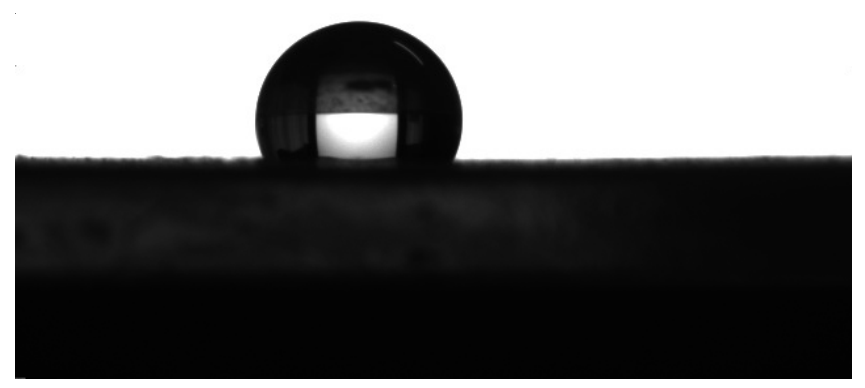

(a)

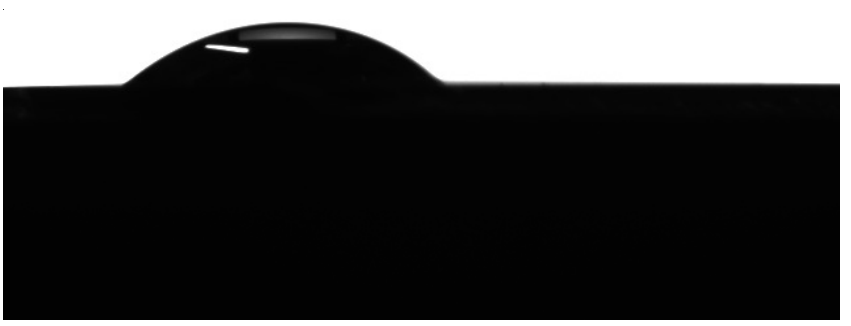

(b)

Fig. 1. Water-contact angles of PDMS (a) and p(SBMA)-PDMS (b)

Protein adsorption properties: BSA was used as a model protein to evaluate the protein resistant characteristics of original and modified PDMS ${ }^{9}$. The samples with the same external surface area were incubated in $1 \mathrm{~g} / \mathrm{L} \mathrm{BSA}$ solutions at $25^{\circ} \mathrm{C}$ for $8 \mathrm{~h}$; $\mathrm{pH}$ was maintained at 7.4 with $0.1 \mathrm{M}$ phosphatebuffered solution (PBS). The coomassie brilliant blue method ${ }^{10}$ was employed to determine BSA concentration using a UVvisible spectrophotometer (Perkin-Elmer, Lambda 35). Measurements of triplicate samples were performed and averaged. The results of BSA adsorption on PDMS and p(SBMA)-PDMS is $c a .1178$ and $454 \mu \mathrm{g} / \mathrm{cm}^{2}$, respectively. It can be seen that BSA adsorption on the membrane surfaces was obviously suppressed after being modified, indicating that introducing $\mathrm{p}$ (SBMA) could improve protein-adsorption-resistance properties 
of PDMS membranes. As was confirmed by Chang et al. ${ }^{11}$ in the aqueous environment the relative high hydrophilicity of $\mathrm{p}$ (SBMA) on modified membrane surface can effectively suppress protein adsorption.

Diatom adsorption properties: Diatom Naricula Parva used in this experiment was cultured by our laboratory as the tested marine fouling biology. When the growth rate of Naricula Parva reached to exponential stage the Naricula Parva was taken out and resuspended on cultures solution to a final concentration of $c a .1 \times 10^{5}$ cells $/ \mathrm{mL}$. Then the PDMS and p(SBMA)-PDMS chips were placed into the Naricula Parva solution and cultured for 7 days at $25^{\circ} \mathrm{C}$. After the experiment the PDMS and p(SBMA)-PDMS chips were taken out and washed by PBS and then was observed by three-dimensional video microscope (HIROX KH-3000, Japan). Fig. 2 shows the results of diatom adsorption on PDMS and p(SBMA)PDMS membrane. It can be seen that after modified with SBMA the PDMS membrane shows excellent diatom adsorption inhibition. The result indicated that introducing $\mathrm{p}$ (SBMA) could improve diatom-adsorption-resistance properties of PDMS membranes.

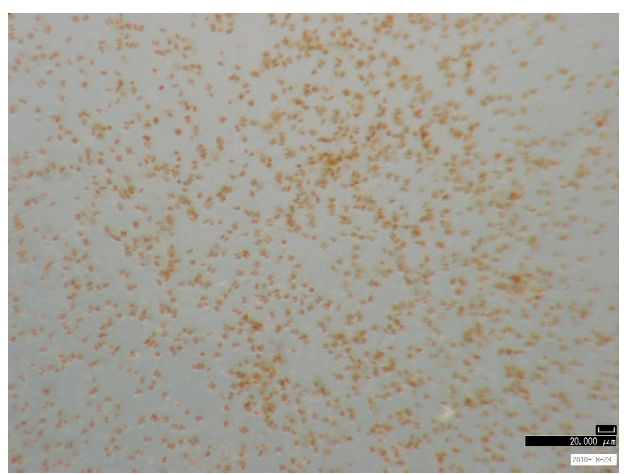

(a)

Fig. 2. Diatom adsorption on PDMS (a) and p(SBMA)-PDMS (b) membrane

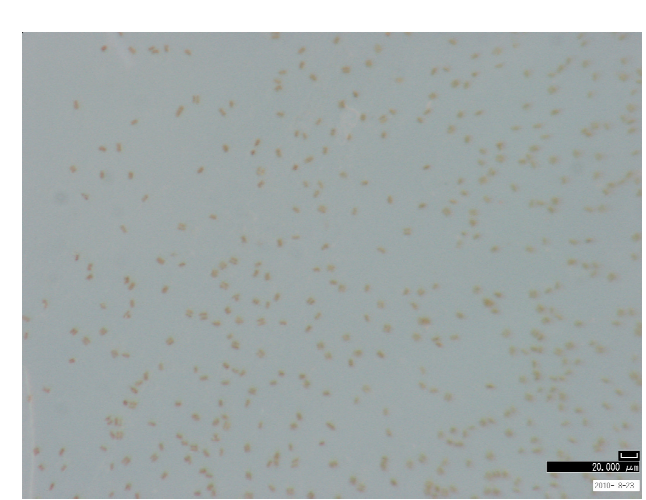

(b)

\section{Conclusion}

$\mathrm{p}$ (SBMA) groups could be introduced onto PDMS membrane surface by grafting via atom transfer radical polymerization (ATRP). ATR/FT-IR spectra results demonstrated the chemical changes occurring on the membrane surface. Results from water-contact angle and bovine serum albumin adsorption measurements revealed that the hydrophilicity of the modified membranes was effectively improved and the protein adsorption of the modified membranes was significantly suppressed. The diatom adsorption experiment revealed that the modified membrane showed excellent diatom inhibition properties. All the above results revealed that the $\mathrm{p}$ (SBMA)-modified PDMS membranes have improved protein-adsorption-resistance and diatom-adsorption-resistance properties and should be potential marine antibiofouling materials and biological and biomedical materials.

\section{REFERENCES}

1. V.V. Papov, V.T. Diamond, K. Biemann and J.H. Waite, J. Biol. Chem., 270, 20183 (1995).

2. M.F.D. Notter, Exp. Cell. Res., 177, 237 (1988).

3. M.P. Olivieri, R.E. Baier and R.E. Loomis, Biomaterials, 13, 1000 (1992).

4. D.C. Hansen, G.W. Luther and J.H. Waite, J. Colloid Interf. Sci., 168, 206 (1994).

5. J.L. Lind, K. Heimann, E.A. Miller, C. van Vliet, N.J. Hoogenraad and R. Wetherbee, Planta, 203, 213 (1997).

6. R.G. Chapman, E. Ostuni, S. Takayama, R.E. Holmlin and L. Yan, G.M. Whitesides, J. Am. Chem. Soc., 122, 8303 (2000).

7. G. Cheng, Z. Zhang, S. Chen, J.D. Bryers and S. Jiang, Biomaterials, 28, 4192 (2007).

8. Y. Chang, S. Liao, A. Higuchi, R. Ruaan, C. Chu and W. Chen, Langmuir, 24, 5453 (2008).

9. J. Zhou, S. Meng, Z. Guo, Q. Du and W. Zhong, J. Membr. Sci., 305, 279 (2007).

10. M.M. Bradford, Anal. Biochem., 72, 248 (1976).

11. Y. Chang, S. Chen, Z. Zhang and S. Jiang, Langmuir, 22, 2222 (2006). 